\section{The $\mathrm{Si}_{3} \mathrm{~N}_{4} / \mathrm{TiN}$ Interface: 1. TiN(001) Grown and Analyzed In situ using Angle-resolved X-ray Photoelectron Spectroscopy}

Richard T. Haasch ${ }^{\text {a) }}$

University of Illinois, Department of Materials Science and Frederick Seitz Materials Research Laboratory, 104 S. Goodwin Avenue, Urbana, IL 61801, USA

Jörg Patscheider

University of Illinois, Department of Materials Science and Frederick Seitz Materials Research Laboratory, 104 S. Goodwin Avenue, Urbana, IL 61801, USA and EMPA, Laboratory for Nanoscale Materials Science, Überlandstrasse 129 CH-8600, Dübendorf, Switzerland

Niklas Hellgren

University of Illinois, Department of Materials Science and Frederick Seitz Materials Research Laboratory, 104 S. Goodwin Avenue, Urbana, IL 61801, USA and Messiah College, Department of Mathematical Sciences, P.O. Box 3041, One College Avenue, Grantham, PA 17027, USA

Ivan Petrov and J. E. Greene

University of Illinois, Department of Materials Science and Frederick Seitz Materials Research Laboratory, 104 S. Goodwin Avenue, Urbana, IL 61801, USA

(Received 11 October 2012; accepted 27 November 2012; published 19 December 2012)

Angle-resolved x-ray photoelectron spectroscopy (AR-XPS) was used to analyze as-deposited epitaxial $\mathrm{TiN}(001)$ layers grown in situ. The films were grown by ultrahigh vacuum reactive magnetron sputtering onto $\mathrm{MgO}(001)$ at $600{ }^{\circ} \mathrm{C}$ in mixed $1: 1 \mathrm{Ar} / \mathrm{N}_{2}$ discharges maintained at a total pressure of $0.5 \mathrm{~Pa}\left(3.75 \times 10^{-3}\right.$ Torr $)$. AR-XPS spectra were obtained using incident monochromatic $\mathrm{Al} K_{\alpha}$ radiation at $0.83401 \mathrm{~nm}$. The results show that the TiN(001) surfaces are free of $\mathrm{O}$ and $\mathrm{C}$. The Ti $2 \mathrm{p}$ photoelectron spectra of clean TiN are characterized by $2 \mathrm{p}_{3 / 2}$ and $2 \mathrm{p}_{1 / 2}$ lines appearing at 454.7 and $460.7 \mathrm{eV}$, each with corresponding satellite lines which are shifted $\sim 2.6 \mathrm{eV}$ to higher binding energies. (C) 2012 American Vacuum Society.

[http://dx.doi.org/10.1116/11.20121001]

Keywords: titanium nitride; magnetron sputtering; hard coating; transition metal nitride

PACS: $81.15 . C d, 61.50 . \mathrm{Lt}, 79.60 . \mathrm{Bm}$

\section{INTRODUCTION}

The bulk properties of nanostructured materials (nanocomposites and nanolaminates) are often defined by the physical and electronic structure of internal interfaces which are not well understood. $\mathrm{Si}_{3} \mathrm{~N}_{4} / \mathrm{TiN}$ nanocomposites (TiN nanocrystals encapsulated in a fully percolated $\mathrm{SiN}_{\mathrm{x}}$ tissue phase) are an example of a class of superhard materials that has attracted much attention as a model system. In previously reported work (Ref. 1), a series of different 4-ML-thick overlayers were deposited by ultrahigh vacuum magnetron sputtering onto epitaxial $\mathrm{TiN}(001)$ on $\mathrm{MgO}(001)$ in order to probe the electronic structure of $\mathrm{Si}_{3} \mathrm{~N}_{4} / \mathrm{TiN}(001), \mathrm{Si} /$ $\mathrm{TiN}(001)$, and $\mathrm{Ti} / \mathrm{TiN}(001)$ bilayer interfaces using angleresolved x-ray photoelectron spectroscopy. The 4-ML overlayer thickness is similar to reported values for the tissue layer in the $\mathrm{Si}_{3} \mathrm{~N}_{4} / \mathrm{TiN}$ nanocomposite system and is also electron transparent, allowing us to probe the electronic structure of the interface. It was found that these three overlayer/TiN(001) interfaces have distinctly different bonding characteristics, significantly influencing the structure of the Ti $2 \mathrm{p}$ photoelectron spectra.

\footnotetext{
a) Author to whom correspondence should be addressed.
}

This contribution presents AR-XPS data from the as-deposited surface of epitaxial $\mathrm{TiN}(001)$, which serves as the base layer for the bilayers listed above, deposited in situ by ultrahigh vacuum reactive magnetron sputtering onto $\mathrm{MgO}(001)$.

\section{SPECIMEN DESCRIPTION (ACCESSION \#01240)}

Host Material: epitaxial TiN(001) thin film

CAS Registry \#: 25583-20-4

Host Material Characteristics: homogeneous; solid; single crystal; conductor; inorganic compound; thin film

Chemical Name: titanium nitride

Source: epitaxially grown in situ on $\mathrm{MgO}(001)$ by ultrahigh vacuum magnetron sputtering

Host Composition: TiN

Form: epitaxial thin film

Structure: $\mathrm{B} 1 \mathrm{NaCl}$ structure

History \& Significance: In order to understand the nature of the $\mathrm{Si}_{3} \mathrm{~N}_{4} / \mathrm{TiN}$ interface, stoichiometric $\mathrm{TiN}(001)$ was first deposited by ultrahigh vacuum (UHV) reactive magnetron sputtering onto $\mathrm{MgO}(001)$ in a growth chamber attached to an analytical 
chamber containing a photoelectron spectrometer. Angleresolved $\mathrm{x}$-ray photoelectron spectra were obtained from asdeposited TiN(001) surfaces without air exposure.

As Received Condition: direct vacuum transfer from growth chamber

Analyzed Region: same as host material

Ex Situ Preparation/Mounting: not specified

In Situ Preparation: Epitaxial TiN(001) layers were grown in a multichamber UHV system. The turbomolecular-pumped deposition chamber, with a base pressure of $<1 \times 10^{-7} \mathrm{~Pa}$ $\left(<1 \times 10^{-9}\right.$ Torr $)$, was equipped with a dc magnetron and isolated from the analytical chamber during growth. $\mathrm{MgO}(001)$ substrates $\left(5 \times 5 \times 0.5 \mathrm{~mm}^{3}\right)$ were annealed at $\mathrm{T}_{\mathrm{s}}=800^{\circ} \mathrm{C}$ for $1 \mathrm{~h}$, a procedure known to produce well-ordered $\mathrm{MgO}(001)$ $1 \times 1$ surfaces as determined by low-energy electron diffraction analyses (Ref. 2). The target, a 5-cm-diameter watercooled Ti (99.9999\%) disc was sputter cleaned in an $\mathrm{Ar} / \mathrm{N}_{2}$ discharge prior to film deposition. The $\mathrm{TiN}(001)$ layers were grown by ultrahigh vacuum reactive magnetron sputter deposition onto $\mathrm{MgO}(001)$ substrates at $600^{\circ} \mathrm{C}$ in mixed $1: 1 \mathrm{Ar} / \mathrm{N}_{2}$ discharges maintained at a total pressure of $0.5 \mathrm{~Pa}(3.75$ $\times 10^{-3}$ Torr). The target power was $100 \mathrm{~W}$ with an electrically floating substrate potential of $7 \mathrm{~V}$. This procedure is known to produce high quality stoichiometric single-crystalline TiN (Ref. 3).

Pre-Analysis Beam Exposure: less than 2 min; no x-ray effects observed

Charge Control: low energy flood gun/magnetic immersion lens combination; filament current $=1.8 \mathrm{~A}$, charge balance $=2.5 \mathrm{~V}$, filament bias $=1 \mathrm{~V}$

Temp. During Analysis: $300 \mathrm{~K}$

Pressure During Analysis: $<3 \times 10^{-7} \mathrm{~Pa}$

INSTRUMENT DESCRIPTION

Manufacturer and Model: Kratos Axis Ultra

Analyzer Type: spherical sector

Detector: channeltron

Number of Detector Elements: 8

INSTRUMENT PARAMETERS COMMON TO ALL SPECTRA -

\section{Spectrometer}

Analyzer Mode: constant pass energy

Throughput $\left(T=E^{N}\right): N=0$

Excitation Source Window: not specified

Excitation Source: $\mathrm{Al} K_{\alpha}$, monochromatic

Source Energy: $1486.6 \mathrm{eV}$

Source Strength: $225 \mathrm{~W}$

Source Beam Size: $2000 \mu \mathrm{m} \times 2000 \mu \mathrm{m}$

Signal Mode: multichannel direct

\section{Geometry}

Incident Angle: varies by spectrum

Source to Analyzer Angle: $54^{\circ}$

Emission Angle: varies by spectrum

Specimen Azimuthal Angle: $45^{\circ}$

Acceptance Angle from Analyzer Axis: $0^{\circ}$

Comments: $\Theta_{\mathrm{e}}=0$ and $\Psi_{\mathrm{i}}=54.7$, (Accession \#s 1240-01 to 05); $\Theta_{\mathrm{e}}=60$ and $\Psi_{\mathrm{i}}=5.3$, (Accession \#s 1240-06 to 10); $\Theta_{\mathrm{e}}=75$ and $\Psi_{\mathrm{i}}=20.3$, (Accession \#s 1240-11 to 15 )

\section{DATA ANALYSIS METHOD}

Energy Scale Correction: The binding energy scale was referenced to the Fermi Level, $E_{f},=0$. The position of $E_{f}$ was estimated by subtracting $1 / 2$ of the full width at half maximum (FWHM) from the position of the maximum of the density-ofstates, DOS, between $0-3 \mathrm{eV}$ as determined by peak fitting (DS(0.2,320)SGL(20)).

Recommended Energy Scale Shift: Accession \#1240-01, 06 and 11, $0 \mathrm{eV}$; Accession \#s 1240-02 to 05, $2.656 \mathrm{eV}$; Accession \#s 1240-07 to 10, $3.032 \mathrm{eV}$; Accession \#s 1240-12 to $15,1.965$ $\mathrm{eV}$

Peak Shape and Background Method: A Shirley background was used (Ref. 4). Asymmetric modified Gaussian/Lorentzian (product formula) line-shapes (Ref. 5 and 6) were used to fit the Ti $2 \mathrm{p}$ and $\mathrm{N} 1 \mathrm{~s}$ spectra.

Quantitation Method: Quantification was done using peak areas from CasaXPS (version 2.3.15) region definitions for the entire peak envelope (main peaks and satellites). For simplicity, the peak amplitudes and concentrations are listed with the lowest binding energy line of each of the different elements. Sensitivity factors supplied by Kratos Analytical.

\section{ACKNOWLEDGMENTS}

This work was was carried out in the Frederick Seitz Materials Research Laboratory Central Facilities, University of Illinois.

\section{REFERENCES}

1. J. Patscheider, N. Hellgren, R. T. Haasch, I. Petrov, and J. E. Greene, Phys. Rev. B 83, 125124 (2011).

2. R. C. Powell, N.-E. Lee, Y.-W. Kim, and J. E. Greene, J. Appl. Phys. 73, 189 (1983).

3. C.-S. Shin, S. Rudenja, D. Gall, N. Hellgren, T.-Y. Lee, I. Petrov, and J. E. Greene, J. Appl. Phys. 95, 356 (2004).

4. D. A. Shirley, Phys. Rev. 55, 4709 (1972).

5. G. Wertheim, J. Electron Spectrosc. 6, 239 (1975).

6. N. Fairley and A. Carrick, The Casa Cookbook Part 1: Recipes for XPS data processing (Acolyte Science, Cheshire, UK, 2005), pp. 147-67.

7. S. Logothetidis, E. I. Meletis, and G. Kouroukis, J. Mater. Res. 14/2, 436 (1999). 


\begin{tabular}{|c|c|c|c|c|c|c|c|}
\hline \multicolumn{8}{|c|}{ SPECTRAL FEATURES TABLE } \\
\hline $\begin{array}{l}\text { Spectrum } \\
\text { ID \# }\end{array}$ & $\begin{array}{l}\text { Element/ } \\
\text { Transition }\end{array}$ & $\begin{array}{c}\text { Peak } \\
\text { Energy } \\
(\mathrm{eV})\end{array}$ & $\begin{array}{c}\text { Peak } \\
\text { Width } \\
\text { FWHM (eV) }\end{array}$ & $\begin{array}{c}\text { Peak } \\
\text { Area } \\
\text { (eV-cts/s) }\end{array}$ & $\begin{array}{l}\text { Sensitivity } \\
\text { Factor }\end{array}$ & $\begin{array}{l}\text { Concentration } \\
\text { (at. \%) }\end{array}$ & $\begin{array}{c}\text { Peak } \\
\text { Assignment }\end{array}$ \\
\hline 01240-02 & $01 \mathrm{~s}$ & 529.7 & 2.5 & 2954.7 & 0.78 & 0.96 & $\cdots$ \\
\hline $01240-03^{a}$ & $\mathrm{Ti} 2 \mathrm{p}_{3 / 2}$ & 454.7 & 0.79 & 431068.6 & 2.001 & 48.63 & TiN \\
\hline $01240-03^{b}$ & $\mathrm{Ti} 2 \mathrm{p}_{3 / 2}$ & 457.5 & 2.74 & $\ldots$ & $\cdots$ & $\cdots$ & TiN \\
\hline $01240-03^{a}$ & $\mathrm{Ti} 2 \mathrm{p}_{1 / 2}$ & 460.7 & 1.03 & $\cdots$ & $\cdots$ & $\cdots$ & TiN \\
\hline $01240-03^{b}$ & $\mathrm{Ti} 2 \mathrm{p}_{1 / 2}$ & 463.6 & 1.37 & $\ldots$ & $\cdots$ & $\cdots$ & TiN \\
\hline 01240-04 & $\mathrm{N} 1 \mathrm{~s}$ & 397 & 0.8 & 94758.5 & 0.477 & 50.4 & TiN \\
\hline $01240-04^{c}$ & $\mathrm{~N} 1 \mathrm{~s}$ & 399.5 & 2.21 & $\cdots$ & $\cdots$ & $\cdots$ & TiN \\
\hline 01240-07 & O 1s & 529.7 & 1.23 & 3301.1 & 0.78 & 2.29 & $\cdots$ \\
\hline 01240-07 & O 1s & 531.2 & 1.38 & $\ldots$ & $\ldots$ & $\ldots$ & $\cdots$ \\
\hline 01240-07 & $01 \mathrm{~s}$ & 533.2 & 1.3 & $\cdots$ & $\ldots$ & $\ldots$ & $\ldots$ \\
\hline $01240-08^{a}$ & $\mathrm{Ti} 2 \mathrm{p}_{3 / 2}$ & 454.8 & 0.85 & 168509.5 & 2.001 & 45.22 & TiN \\
\hline $01240-08^{b}$ & $\mathrm{Ti} 2 \mathrm{p}_{3 / 2}$ & 457.6 & 2.55 & $\ldots$ & $\ldots$ & $\ldots$ & TiN \\
\hline $01240-08^{a}$ & $\mathrm{Ti} 2 \mathrm{p}_{1 / 2}$ & 460.8 & 1.11 & $\cdots$ & $\cdots$ & $\cdots$ & TiN \\
\hline $01240-08^{b}$ & $\mathrm{Ti} 2 \mathrm{p}_{1 / 2}$ & 463.7 & 1.5 & $\ldots$ & $\ldots$ & $\ldots$ & TiN \\
\hline 01240-09 & N 1s & 397.1 & 0.95 & 46050.1 & 0.477 & 52.19 & TiN \\
\hline $01240-09^{c}$ & $\mathrm{~N} 1 \mathrm{~s}$ & 399.6 & 2.18 & $\ldots$ & $\ldots$ & $\ldots$ & TiN \\
\hline 01240-12 & O 1s & 530.3 & 1.89 & 3656.4 & 0.78 & 4.51 & $\ldots$ \\
\hline 01240-12 & $01 \mathrm{~s}$ & 532 & 2 & $\ldots$ & $\cdots$ & $\cdots$ & $\cdots$ \\
\hline 01240-12 & $01 \mathrm{~s}$ & 533.9 & 2 & $\cdots$ & $\cdots$ & $\cdots$ & $\cdots$ \\
\hline $01240-13^{a}$ & $\mathrm{Ti} 2 p_{3 / 2}$ & 454.9 & 0.9 & 96816.6 & 2.001 & 46.6 & TiN \\
\hline $01240-13^{b}$ & $\mathrm{Ti} 2 \mathrm{p}_{3 / 2}$ & 457.6 & 2.57 & $\ldots$ & $\ldots$ & $\ldots$ & TiN \\
\hline $01240-13^{a}$ & $\mathrm{Ti} 2 \mathrm{p}_{1 / 2}$ & 460.9 & 1.16 & $\cdots$ & $\cdots$ & $\ldots$ & TiN \\
\hline $01240-13^{b}$ & $\mathrm{Ti} 2 \mathrm{p}_{1 / 2}$ & 463.7 & 1.5 & $\ldots$ & $\cdots$ & $\cdots$ & TiN \\
\hline 01240-14 & N 1s & 397.1 & 1.04 & 24245.9 & 0.477 & 48.9 & TiN \\
\hline $01240-14^{c}$ & $\mathrm{~N} 1 \mathrm{~s}$ & 399.6 & 1.59 & $\ldots$ & $\ldots$ & $\ldots$ & TiN \\
\hline
\end{tabular}

a screened final-state

b unscreened final-state, plasmon (Ref. 7)

c plasmon (Ref. 7)

Comment to Spectral Features Table: Quantification was done using peak areas from CasaXPS (version 2.3.15) region definitions for the entire peak envelope (main peaks and satellites). For simplicity, the peak amplitudes and concentrations are listed with the lowest binding energy line of each of the different elements. Sensitivity factors supplied by Kratos Analytical. 


\begin{tabular}{|c|c|c|c|c|c|}
\hline \multicolumn{6}{|c|}{ GUIDE TO FIGURES } \\
\hline $\begin{array}{l}\text { Spectrum } \\
\text { (Accession) \# }\end{array}$ & $\begin{array}{l}\text { Spectral } \\
\text { Region }\end{array}$ & $\begin{array}{c}\text { Voltage } \\
\text { Shift }^{\star}\end{array}$ & Multiplier & Baseline & Comment \# \\
\hline $1240-01$ & survey & 0 & 1.00 & 1000000 & 1 \\
\hline $1240-02$ & O 1s & -2.656 & 1.00 & 0 & 1 \\
\hline $1240-03$ & Ti $2 p$ & -2.656 & 1.00 & 11000 & 1 \\
\hline $1240-04$ & $\mathrm{~N} 1 \mathrm{~s}$ & -2.656 & 1.00 & 18000 & 1 \\
\hline $1240-05$ & valence band & -2.656 & 1.00 & 400 & 1 \\
\hline $1240-06$ & survey & 0 & 3.41 & 500000 & 2 \\
\hline $1240-07$ & $01 \mathrm{~s}$ & -3.032 & 1.02 & 4000 & 2 \\
\hline $1240-08$ & Ti $2 p$ & -3.032 & 2.41 & 5000 & 2 \\
\hline $1240-09$ & $\mathrm{~N} 1 \mathrm{~s}$ & -3.032 & 2.49 & 8000 & 2 \\
\hline $1240-10$ & valence band & -3.032 & 2.41 & 200 & 2 \\
\hline $1240-11$ & survey & 0 & 5.74 & 0 & 3 \\
\hline $1240-12$ & $01 \mathrm{~s}$ & -1.965 & 1.04 & 4700 & 3 \\
\hline $1240-13$ & Ti $2 p$ & -1.965 & 4.35 & 0 & 3 \\
\hline $1240-14$ & $\mathrm{~N} 1 \mathrm{~s}$ & -1.965 & 4.87 & 0 & 3 \\
\hline $1240-15$ & valence band & -1.965 & 4.20 & 0 & 3 \\
\hline
\end{tabular}

*Voltage shift of the archived (as-measured) spectrum relative to the printed figure. The figure reflects the recommended energy scale correction due to a calibration correction, sample charging, flood gun, or other phenomenon.

"*NP] signifies not published; digital spectra are archived in SSS database but not reproduced in the printed journal.

1. $\Theta_{\mathrm{e}}=0^{\circ}$

2. $\Theta_{\mathrm{e}}=60^{\circ}$

3. $\Theta_{\mathrm{e}}=75^{\circ}$ 


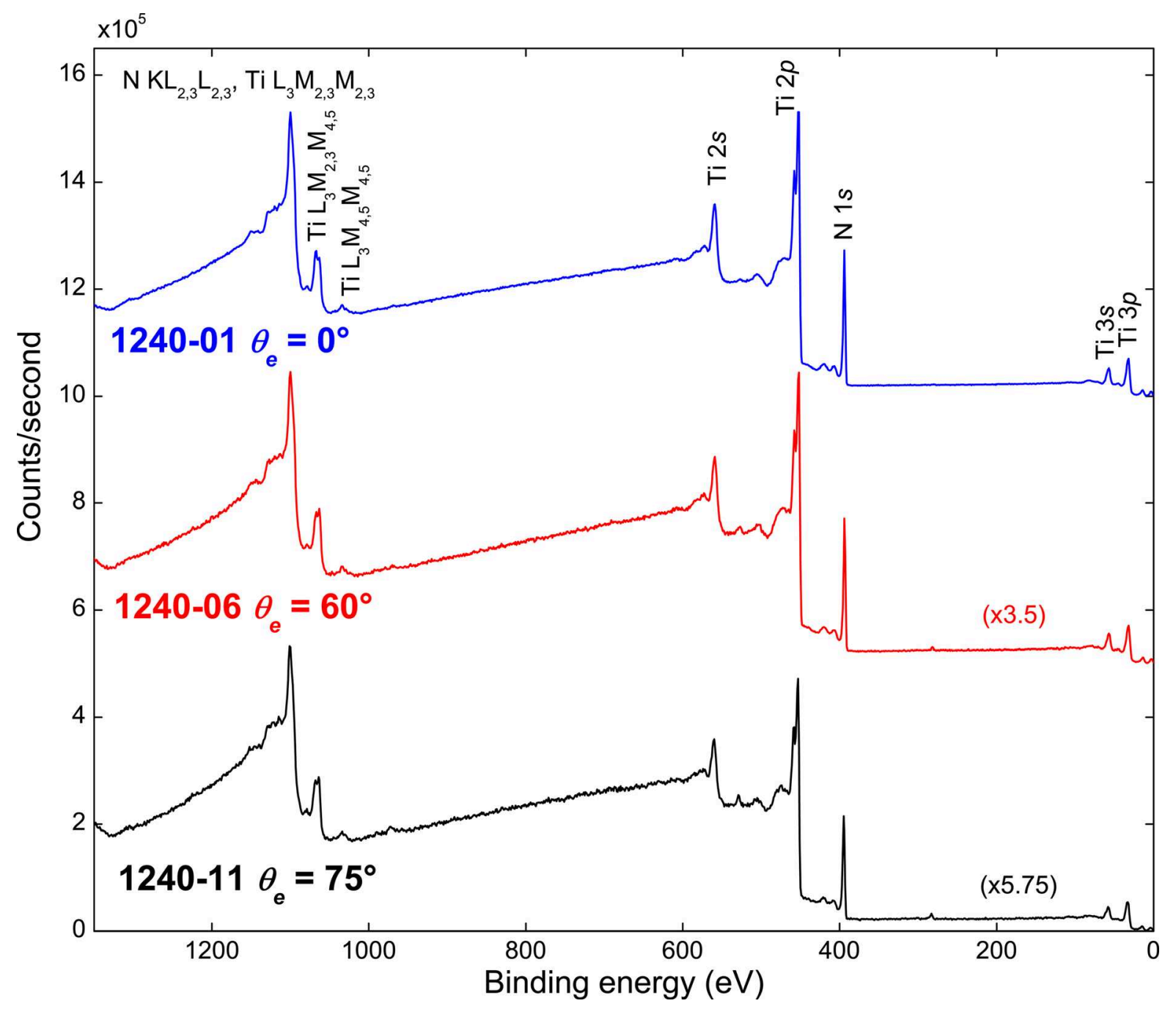

\begin{tabular}{|c|c|}
\hline Accession \# & $01240-01,01240-06,01240-11$ \\
\hline Host Material & epitaxial TiN(001) thin film \\
\hline Technique & XPS \\
\hline Spectral Region & survey \\
\hline Instrument & Kratos Axis Ultra \\
\hline Excitation Source & Al $K_{\alpha}$ monochromatic \\
\hline Source Energy & $1486.6 \mathrm{eV}$ \\
\hline Source Strength & $225 \mathrm{~W}$ \\
\hline Source Size & $2 \mathrm{~mm} \times 2 \mathrm{~mm}$ \\
\hline Analyzer Type & spherical sector \\
\hline Incident Angle & varies by spectrum \\
\hline Emission Angle & varies by spectrum \\
\hline Analyzer Pass Energy: & $160 \mathrm{eV}$ \\
\hline Analyzer Resolution & $2.4 \mathrm{eV}$ \\
\hline Total Signal Accumulation Time & $270 \mathrm{~s}$ \\
\hline Total Elapsed Time & \#01240-01: 530 s; \#01240-06: 530 s; \#01240-11: 480 s \\
\hline Number of Scans & 2 \\
\hline Effective Detector Width & $33.6 \mathrm{eV}$ \\
\hline Comment & $\begin{array}{c}\# 01240-01: \text { emission angle }=0^{\circ} ; \# 01240-06: \text { emission angle }=60^{\circ} ; \\
\# 01240-11: \text { emission angle }=75^{\circ}\end{array}$ \\
\hline
\end{tabular}




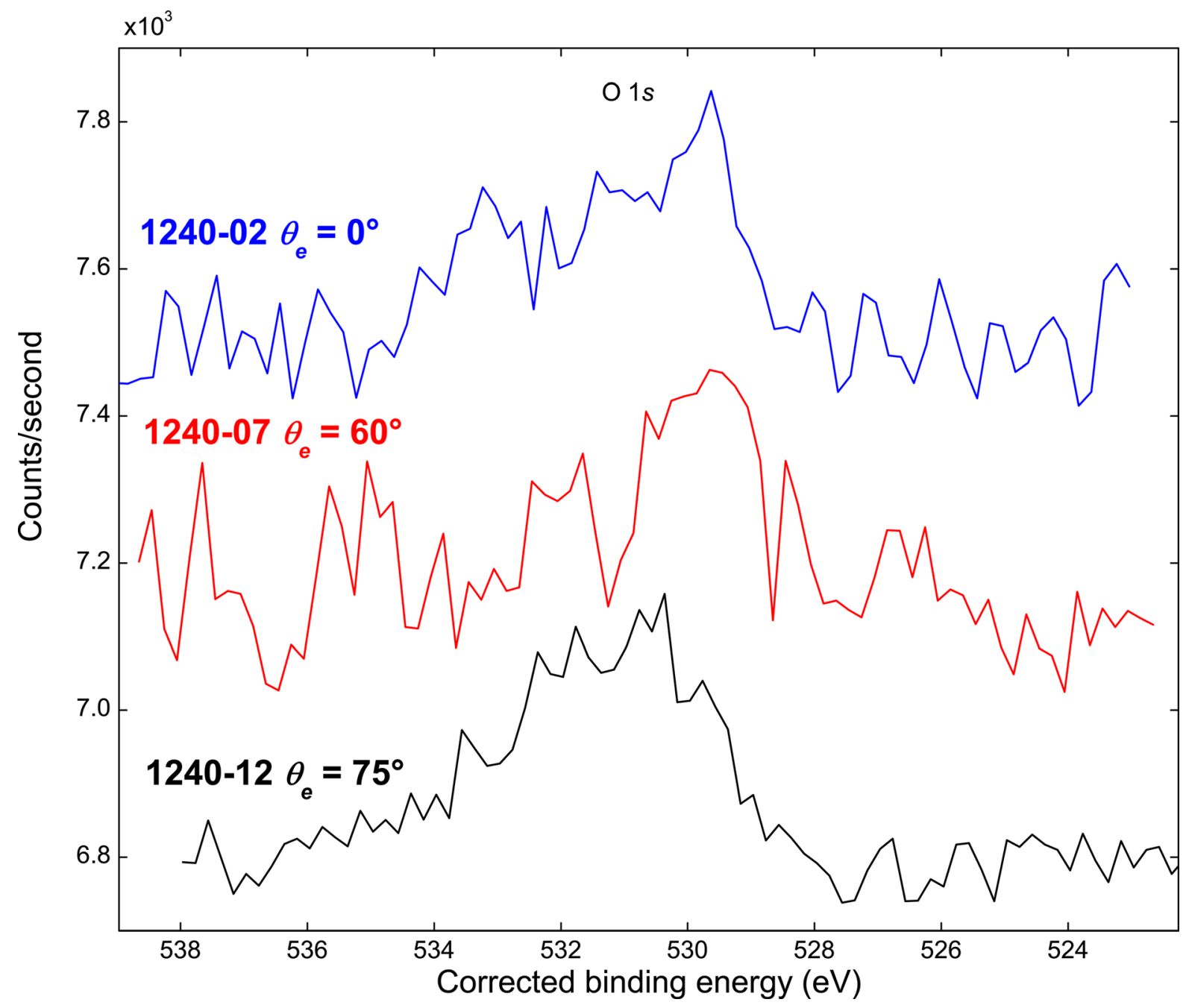

\begin{tabular}{rc}
\hline Accession $\#$ & $\mathbf{0 1 2 4 0 - 0 2 , 0 1 2 4 0 - 0 7 , 0 1 2 4 0 - 1 2}$ \\
Host Material & epitaxial TiN(001) thin film \\
Technique & XPS \\
Spectral Region & O 1s \\
Instrument & Kratos Axis Ultra \\
Excitation Source & Al $K_{\alpha}$ monochromatic \\
Source Energy & $1486.6 \mathrm{eV}$ \\
Source Strength & $225 \mathrm{~W}$ \\
Source Size & 2 mm $\times 2$ mm \\
Analyzer Type & spherical sector \\
Incident Angle & varies by spectrum \\
Emission Angle & varies by spectrum \\
Analyzer Pass Energy: & $20 \mathrm{eV}$ \\
Analyzer Resolution & $0.3 \mathrm{eV}$ \\
Total Elapsed Time & $\# 01240-02: 81 \mathrm{~s} ; \# 01240-07: 81 \mathrm{~s} ; \# 01240-12: 122 \mathrm{~s}$ \\
Number of Scans & $\# 01240-02: 223 \mathrm{~s} ; 01240-07: 223 \mathrm{~s} ; \# 01240-12: 335 \mathrm{~s}$ \\
Total Signal Accumulation Time & $\# 01240-02: 10 ; \# 01240-07: 10 ; \# 01240-12: 15$ \\
Comment & $4.2 \mathrm{eV}$ \\
& $\# 01240-02:$ emission angle $=0^{\circ} ; \# 01240-07:$ emission angle $=60^{\circ} ;$ \\
\hline
\end{tabular}




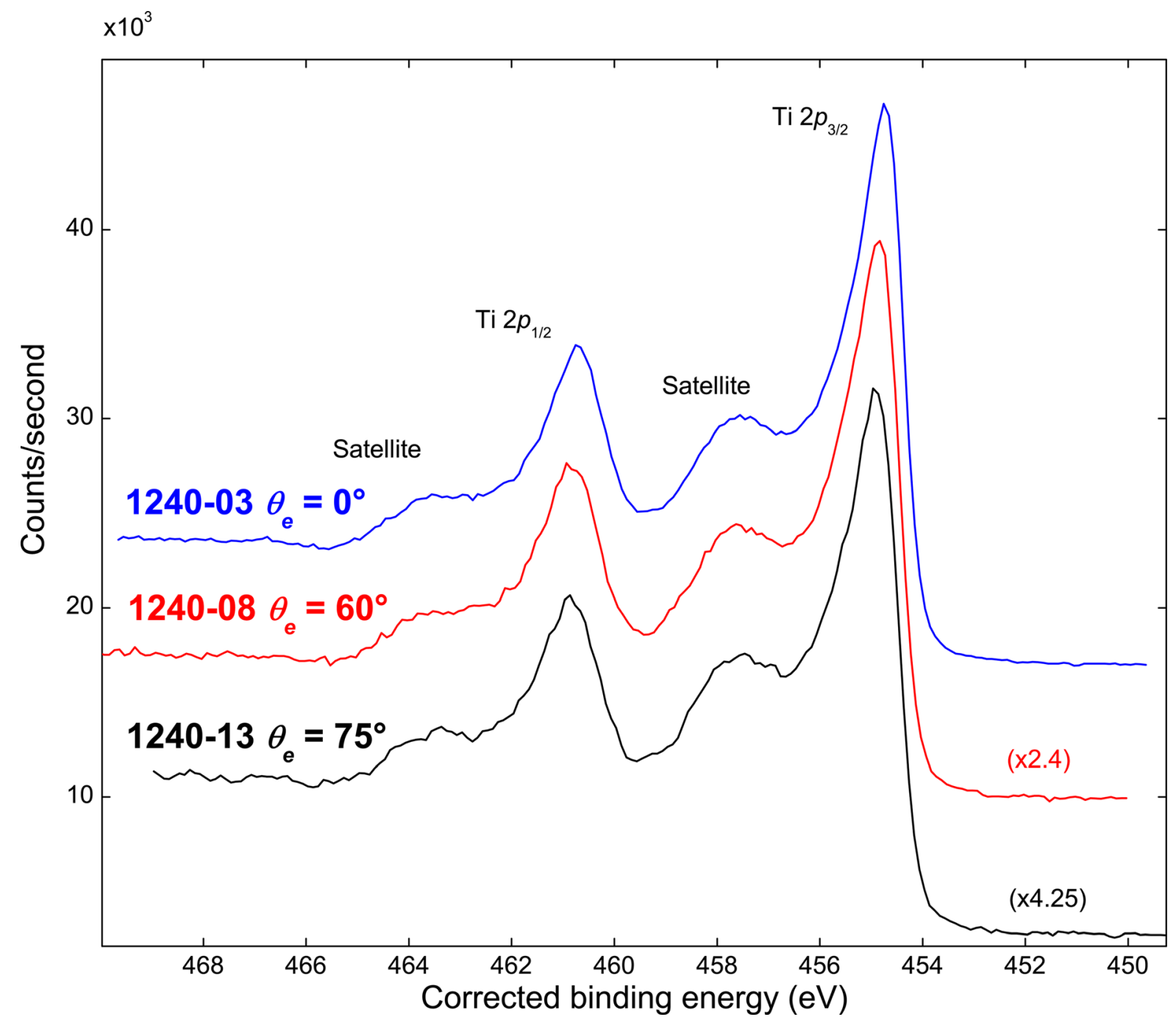

\begin{tabular}{rc}
\hline Accession \# & 01240-03, 01240-08, 01240-13 \\
Host Material & epitaxial TiN(001) thin film \\
Sechnique & XPS \\
Instrument & Ti 2p \\
Excitation Source & Kratos Axis Ultra \\
Source Energy & Al $K_{\alpha}$ monochromatic \\
Source Strength & $1486.6 \mathrm{eV}$ \\
Source Size & $225 \mathrm{~W}$ \\
Analyzer Type & 2 mm $\times 2$ mm \\
Incident Angle & spherical sector \\
Emission Angle & varies by spectrum \\
Analyzer Pass Energy: & varies by spectrum \\
Analyzer Resolution & 20 eV \\
Total Elapsed Time & $0.3 \mathrm{eV}$ \\
Number of Scans & $\# 01240-03: 201 \mathrm{~s} ; \# 01240-08: 201 \mathrm{~s} ; \# 01240-13: 302 \mathrm{~s}$ \\
Total Signal & $\# 01240-03: 555 \mathrm{~s} ; \# 01240-08: 555 \mathrm{~s} ; 01240-13: 832 \mathrm{~s}$ \\
Effective Detector Width & $\# 01240-03: 10 ; \# 01240-08: 10 ; \# 01240-13: 15$ \\
Comment & $4.2 \mathrm{eV}$
\end{tabular}




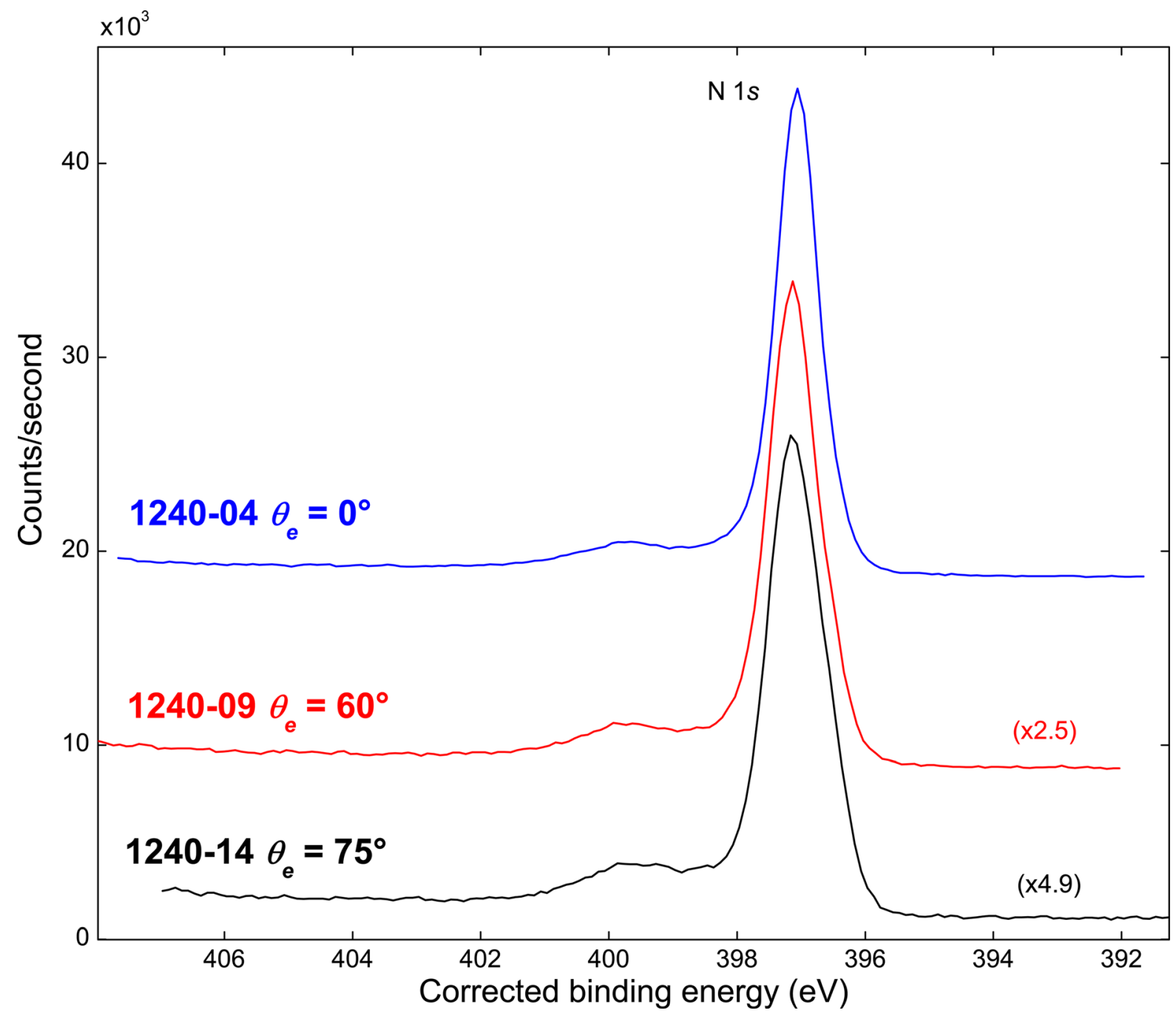

Accession \#

01240-04, 01240-09, 01240-14

Host Material

Technique

Spectral Region

Instrument

Excitation Source

Source Energy

Source Strength

Source Size

Analyzer Type

Incident Angle

Emission Angle

Analyzer Pass Energy:

Analyzer Resolution

Total Signal Accumulation Time

Total Elapsed Time

Number of Scans

Effective Detector Width

Comment epitaxial TiN(001) thin film

$$
\text { XPS }
$$

$\mathrm{N} 1 \mathrm{~s}$

Kratos Axis Ultra

Al $K_{\alpha}$ monochromatic

$1486.6 \mathrm{eV}$

$225 \mathrm{~W}$

$2 \mathrm{~mm} \times 2 \mathrm{~mm}$

spherical sector

varies by spectrum

varies by spectrum

$$
\begin{aligned}
& 20 \mathrm{eV} \\
& 0.3 \mathrm{eV}
\end{aligned}
$$

\#01240-04: 161 s; \#01240-09: 161 s; \#01240-14: $242 \mathrm{~s}$

\#01240-04: 444 s; \#01240-09: 444 s; \#01240-14: $666 \mathrm{~s}$ \#01240-04: 10; \#01240-09: 10; \#01240-14: 15

$$
4.2 \mathrm{eV}
$$

$\# 01240-04:$ emission angle $=0^{\circ} ; \# 01240-09$ : emission angle $=60^{\circ}$; $\# 01240-14$ : emission angle $=75^{\circ}$ 


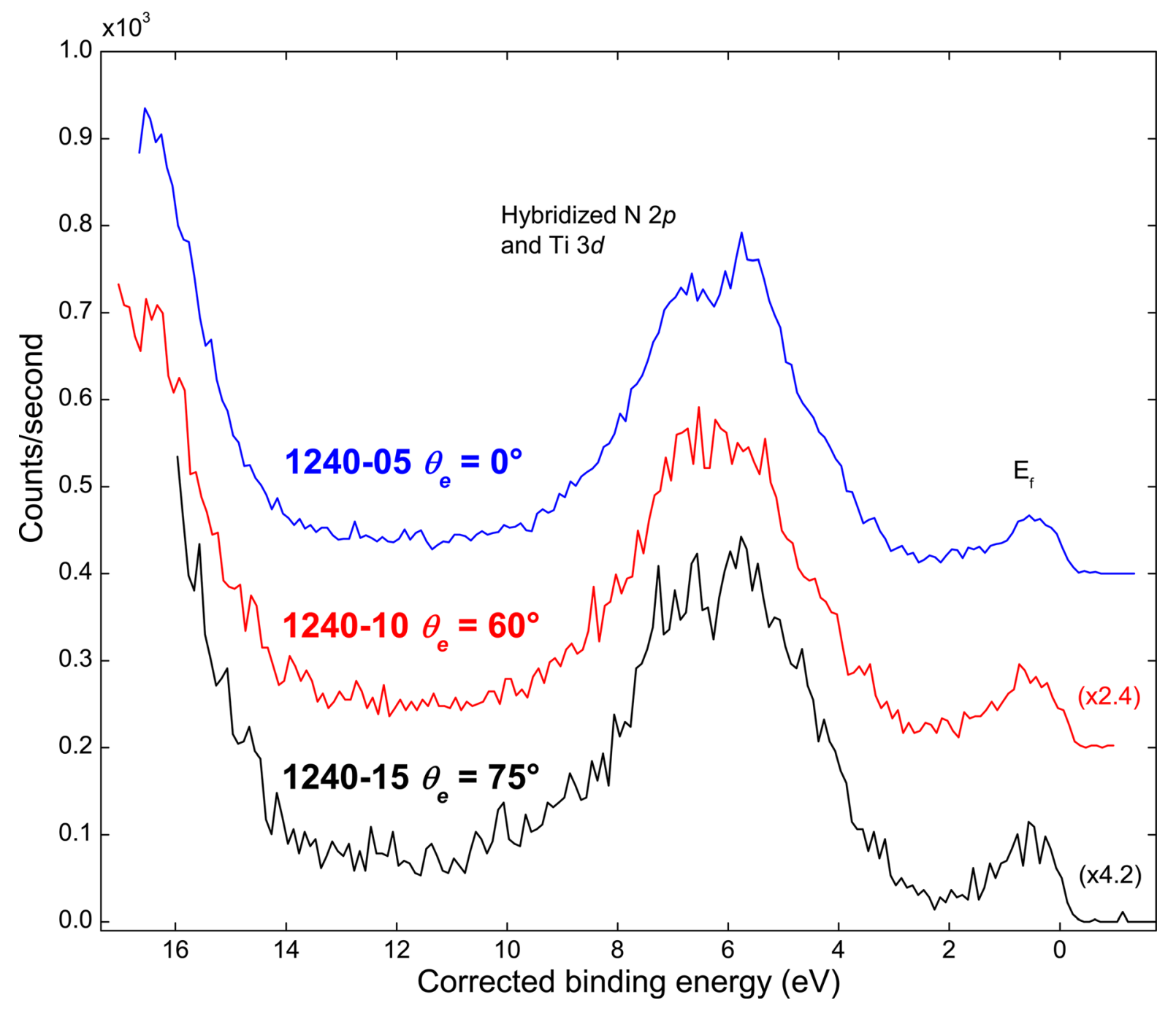

\begin{tabular}{|c|c|}
\hline Accession \# & 01240-05, 01240-10, 01240-15 \\
\hline Host Material & epitaxial TiN(001) thin film \\
\hline Technique & XPS \\
\hline Spectral Region & valence band \\
\hline Instrument & Kratos Axis Ultra \\
\hline Excitation Source & Al $K_{\alpha}$ monochromatic \\
\hline Source Energy & $1486.6 \mathrm{eV}$ \\
\hline Source Strength & $225 \mathrm{~W}$ \\
\hline Source Size & $2 \mathrm{~mm} \times 2 \mathrm{~mm}$ \\
\hline Analyzer Type & spherical sector \\
\hline Incident Angle & varies by spectrum \\
\hline Emission Angle & varies by spectrum \\
\hline Analyzer Pass Energy: & $20 \mathrm{eV}$ \\
\hline Analyzer Resolution & $0.3 \mathrm{eV}$ \\
\hline Total Signal Accumulation Time & \#01240-05: 181 s; \#01240-10: 181 s; \#01240-15: 272 s \\
\hline Total Elapsed Time & \#01240-05: 499 s; \#01240-10: 499 s; \#01240-15: 749 s \\
\hline Number of Scans & \#01240-05: 10; \#01240-10: 10; \#01240-15: 15 \\
\hline Effective Detector Width & $4.2 \mathrm{eV}$ \\
\hline Comment & $\begin{array}{l}\# 01240-05: \text { emission angle }=0^{\circ} ; \# 01240-10: \text { emission angle }=60^{\circ} ; \\
\# 01240-15: \text { emission angle }=75^{\circ}\end{array}$ \\
\hline
\end{tabular}

